\title{
Comparison of two earthquake early warning location methods
}

\author{
Jun Li $\cdot$ Xing Jin $\cdot$ Hongcai Zhang $\cdot$ \\ Yongxiang Wei
}

Received: 11 May 2012/Accepted: 12 March 2013/Published online: 2 November 2013

(C) The Seismological Society of China, Institute of Geophysics, China Earthquake Administration, and Springer-Verlag Berlin Heidelberg 2013

\begin{abstract}
According to earthquake catalog records of Fujian Seismic Network, the $T_{\text {now }}$ method and the fourstation continuous location method put forward by Jin Xing are inspected by using P-wave arrival information of the first four stations in each earthquake. It shows that the fourstation continuous location method can locate more seismic events than the $T_{\text {now }}$ method. By analyzing the results, it is concluded that the reason for this is that the $T_{\text {now }}$ method makes use of information from stations without being triggered, while some stations failed to be reflected in earthquake catalog because of discontinuous records or unclear records of seismic phases. For seismic events whose location results can be given, there is no obvious difference in location results of the two methods and positioning deviation of most seismic events is also not significant. For earthquakes outside the network, the positioning deviation may amplify as the epicentral distance enlarges, which may relate to the situation that the seismic stations are centered on one side of epicenter and the opening angle between seismic stations used for location and epicenter is small.
\end{abstract}

\footnotetext{
J. Li $(\bowtie) \cdot$ X. Jin $\cdot$ H. Zhang $\cdot$ Y. Wei

Earthquake Administration of Fujian Province, Fuzhou 350003,

China

e-mail: lixjix@163.com

X. Jin

Institute of Engineering Mechanics, China Earthquake

Administration, Harbin 150080, China

X. Jin

Fuzhou University, Fuzhou 350002, China
}

Keywords Earthquake early warning $\cdot T_{\text {now }}$ location method $\cdot$ Earthquake catalog $\cdot$ Four-station continuous location method

\section{Introduction}

Earthquake location is a fundamental problem in seismology, and its main task is to determine the earthquake location (epicenter location and focal depth) and the origin time accurately. However, rapid and reliable location in the earthquake early warning is a primary issue which decides whether the earthquake early warning is successful or not. Now, it is difficult for the frequently used traditional earthquake location methods to meet the requirement for timeliness. For this, a variety of fast location methods have been developed in China and abroad. Nakamura et al. (1988), Nakamura and Tucker (1988), and Nakamura (1989) put forward a method, which uses the horizontal amplitude ratio after level and smooth and vertical symbol to determine azimuth of epicenter after the P-wave's arrival, estimates the earthquake magnitude by P-wave's predominant frequency, and determines the epicentral distance by the attenuation relationship among the amplitude of the initial information and magnitude and distance. The method belongs to empirical methods and uses the magnitude which is derived by the estimation of P-wave's predominant frequency in the process of location, which induces large location errors. Although the method has great limitations, its successful application in the UrEDAS system of Japan has promoted the development of earthquake early warning technology. Odaka et al. (2003) used the exponential function $B t \cdot \exp (-A t)$ to fit the absolute value recorded by vertical $\mathrm{P}$-wave in the first $3 \mathrm{~s}$. In the exponential function, $t$ is the $\mathrm{P}$-wave arrival time, and $A$ and $B$ are the parameters 
which need fitting. It is considered that $B$ value is related to the epicentral distance. Adding it and the epicenter azimuth, the method can locate the epicenter. However, it also belongs to empirical method, which will lead to large location errors as well. Wu and Teng (2002) applied strong earthquake network in Taiwan to build a virtual subnet, and used a traditional single-layer crustal velocity model method to locate, which was less time-sensitive but had large blind zone in the early warning so that it could only be applied to remote early warning. Rydelek and Pujol (2004) developed a double sub-station location method, which could limit the epicenter to one of the hyperbolas according to the different arrival time of P-wave, use location information about other stations location without being triggered, and restrict the epicenter to one section of the hyperbolas. Based on Rydelek's research, Horiuchi et al. (2005) used current time $T_{\text {now }}$ to define the station whether it was triggered or not and determined the point with the minimum residual as the epicenter by a grid search method. In China, Jin et al. (2012) fully considered the current situation and future development trend of domestic seismic network and put forward the four-station continuous location method which re-derived and simplified the earthquake location formula and realized continuous correction made by information update of location results.

According to comparative analysis between the $T_{\text {now }}$ method and the real-time continuous location method put forward by Jin et al. (2012), we can conclude that both methods adopt the "Voronoi triangulation (Satriano et al. 2008) location when a single station is triggered, the region which does not meet the trigger condition will be excluded gradually as the time process continues. The epicenter can be limited to a section of the hyperbola when the double stations ar triggered; similarly, as the time process continues, the region which does not meet the trigger condition will be excluded gradually. In general, because of the comparatively small amount of information available, the location results by using single or double station to locate earthquakes have several deviations. When there are more than three or four stations that are triggered, the location results have higher reliability. This thesis describes the inspection and comparative analysis of the four-station location results of the two kinds of earthquake early warning location methods and possible problems in the location process according to the earthquake catalog of Fujian Seismic Network.

\section{Methods and data}

\section{$2.1 T_{\text {now }}$ method introduction}

It is assumed that a large earthquake occurs and two stations detect $\mathrm{P}$-wave arrival before $T_{\text {now }}$, while the other stations have not yet detected the $\mathrm{P}$ arrival. The $\mathrm{P}$-wave arrival time for the two stations restricts the permissible epicenter location to a certain place along a hyperbolic curve. In addition, we assume that this earthquake is sufficiently large such that the other stations will detect seismic signals later so long as they are operating properly. Therefore, this event occurs at a time and in an area such that all the stations-except for the first two stations which detect the arrival-are unable to detect $\mathrm{P}$-waves before $T_{\text {now. }}$. An inequality equation can be written for the stations without the P-wave arrival since the theoretic arrival time at the stations is larger than that of $T_{\text {now }}$.

$T^{\text {now }}-T_{i}^{\text {delay }}-T_{h i}(\varphi, \lambda, h, t)<0$,

where $T_{h i}$ is the theoretic arrival time of the $\mathrm{P}$-wave at the $i$ th station, and $\varphi, \lambda, h$, and $t$ are the latitude, longitude, focal depth, and origin time of the event, respectively. $T_{i}^{\text {delay }}$ is the total (known) amount of time delay in the transmission of the waveform data from the $i$ th station to the computer in the automatic processing system.

Residuals for arrival time data can be expressed by

$R_{\mathrm{p} j}=T_{i}^{\mathrm{p}}-T_{h j}(\varphi, \lambda, h, t)$,

where $T_{j}^{\mathrm{p}}$ is the observed $\mathrm{P}$-wave arrival time for the $j$ th station. Define residuals for $T_{\text {now }}$ as follows.

$$
\begin{aligned}
& R_{n i}=T^{\text {now }}-T_{i}^{\text {delay }}-T_{h i}(\varphi, \lambda, h, t) \\
& \quad T_{h i}+T_{i}^{\text {delay }}-T^{\text {now }}<\varepsilon_{0} \\
& R_{n i}=-\varepsilon(\Delta, h) ; \quad T_{h i}+T_{i}^{\text {delay }}-T^{\text {now }} \geq \varepsilon_{0},
\end{aligned}
$$

where $\varepsilon_{0}$ is a some positive small constant and $\varepsilon(\Delta, h)$ is a simple positive function that depends on both hypocentral depth $h$ and epicentral distance $\Delta$,

$$
\begin{aligned}
\varepsilon(\Delta, h) & =a\left|h-h_{0}\right|+b \Delta+\varepsilon_{0} & & N_{\mathrm{p}}=2,3 \\
& =\varepsilon_{0} & & N_{\mathrm{p}}=4
\end{aligned}
$$

$a, h_{0}$, and $b$ are constants with pre-assigned values and $N_{\mathrm{p}}$ is the number of P-wave arrival time. Since far and deep events have larger apparent velocities than those for local shallow events, there will be more stations observing P-wave arrivals within a certain time interval measured from the initial $\mathrm{P}$-wave arrival at the close station. According to the past experience, assume $h_{0}, a$, and $b$ to be $10 \mathrm{~km}, 0.01 \mathrm{~s} / \mathrm{km}$, and $0.01 \mathrm{~s} / \mathrm{km}$, respectively.

The hypocenter location is determined by minimizing the sum of squares of residuals:

$\rho^{2}=\sum_{j} W_{\mathrm{p} j} R_{\mathrm{p} j}^{2}+\sum_{i} W_{n i} R_{n i}^{2}$,

where $W_{\mathrm{p} j}$ and $W_{n i}$ are weights for the P-wave arrival time and for $T_{\text {now }}$, respectively. For the purpose of calculations, we assume the weight for $W_{n i}$ to be $1 / 20$ of $W_{\mathrm{p} j}$. The 
theoretic arrival time is calculated by using a velocity model that consists of two layers having a vertical velocity gradient (Hasegawa et al. 1978).

The initial location of the earthquake's hypocenter is put at a depth of $50 \mathrm{~km}$ beneath the station with the first $\mathrm{P}$-wave arrival. The origin time for the event is always computed from the estimated location and the velocity structure in our model. Surrounding this location, we construct a three-dimensional grid $(3 \times 3 \times 3=27$ elements) with a given grid spacing such that the center of the grid coincides with the initial hypocentral location. The residuals from Eq. (6) are calculated at each point of the grid. When a minimum value is found for the grid, we then shift the location of the entire three-dimensional grid so that the center of the new grid coincides with the location of this minimum value. This procedure is repeated until the center of the new grid becomes the point of the minimum value of Eq. (6). The grid intervals are then decreased by one-third of the original length, and Eq. (6) is recalculated until a new minimum is similarly obtained for the smaller grid. This procedure is iterated until the final grid length becomes less than $1 \mathrm{~km}$.

\subsection{Introduction to the four-station continuous location method}

Based on the basic law of earthquake spread, this method derived a set of condensed formulas to locate by taking advantage of the P-wave arrival information of the first four trigger stations, and they are shown as follows: the formula derivation adopts the local coordinate system which uses the first trigger station $S_{1}$ as the origin of coordinates, connects the first triggered station $S_{1}$ to the second trigger station $S_{2}$ as the positive direction of $X$-axis of the local coordinate system, and determines the positive direction of $Y$-axis according to the right-hand rule. At this time, positions of the first three trigger stations within the local coordinate system is $(0,0),\left(d_{12}, 0\right)$, and $\left(x_{3}, y_{3}\right)$, respectively. $d_{12}$ indicates the distance between $S_{1}$ and $S_{2}$ stations, $x_{3}, y_{3}$ is the coordinates of the station $S_{3}$ after the coordinate transformation. We assume the wave velocity is locally uniform, P-wave's velocity is constantly $V_{\mathrm{p}}$. Then, the difference in the focal distance from the second station and the third station to the first station is shown as follows.

$\left\{\begin{array}{l}S_{21}=r_{2}-r_{1}=V_{\mathrm{p}}\left(T_{\mathrm{p} 2}-T_{\mathrm{p} 1}\right)=V_{\mathrm{p}} t_{21} \\ S_{31}=r_{3}-r_{1}=V_{\mathrm{p}}\left(T_{\mathrm{p} 3}-T_{\mathrm{p} 1}\right)=V_{\mathrm{p}} t_{31}\end{array}\right.$.

In the formula, $t_{21}$ and $t_{31}$ are the arrival time difference of the first triggered station $S_{1}$ to the second and the third triggered stations, respectively. Bring the epicentral distance and focal distance into the above equation and simplify it (the specific derivation process is available in Jin et al. 2012), it can be concluded that: $\left\{\begin{array}{l}x=p_{1}+p_{2} r_{1} \\ y=b_{1}+b_{2} r_{1}\end{array}\right.$

The expressions of the parameters $p_{1}, p_{2}, b_{1}$, and $b_{2}$ are shown as follows:

$\left\{\begin{array}{l}p_{1}=\frac{1}{2 d_{12}}\left(d_{12}^{2}-S_{21}^{2}\right) \\ p_{2}=-\frac{S_{21}}{d_{12}} \\ b_{1}=\frac{1}{2 y_{3}}\left(d_{13}^{2}-2 x_{3} p_{1}-S_{31}^{2}\right) \\ b_{2}=-\frac{1}{y_{3}}\left(x_{3} p_{2}+S_{31}\right)\end{array}\right.$

In the formula, $d_{13}$ is the distance of the first triggered station $S_{1}$ to the third station. The epicenter location coordinates can be written as a parametric equation relevant to the focal distance $r_{1}$ of the first trigger station $S_{1}$, which is a linear equation; namely, with three P-wave arrival information, the epicenter can be limited to a straight line segment with finite length:

$y=b_{1}+\frac{b_{2}\left(x-p_{1}\right)}{p_{2}}=\left(b_{1}-\frac{b_{2} p_{1}}{p_{2}}\right)+\frac{b_{2}}{p_{2}} x$,

In fact, only the P-wave arrival information of the first three stations cannot accurately calculate the focal distance. At that time, we can only limit the possible epicenter location to a straight line segment. Although the above equation appears to be a straight line equation, it is a space curve containing the focal depth. If we assume that the focal depth is acquired, the epicentral location can be calculated.

When the P-wave arrival information of the fourth station is picked up (local coordinates are $x_{4}, y_{4}$ ), the parameter $r_{1}$ can be calculated as follows:

$r_{1}=\frac{1}{2} \frac{\left[d_{14}^{2}-S_{41}^{2}-2\left(x_{4} p_{1}+y_{4} b_{1}\right)+z_{4}^{2}-2 h z_{4}\right]}{\left[S_{41}+x_{4} p_{2}+y_{4} b_{2}\right]}$,

where $x_{4}, y_{4}$ is local coordinates of the fourth station and $d_{14}$ is the distance from the first triggered station $S_{1}$ to the fourth triggered station. According to Eq. (8), the epicenter location can be accurately estimated.

\subsection{The selection of seismic events}

The earthquake early warning mainly has obvious effect on major earthquakes, while the number of major earthquakes is small, and the number of the major earthquakes with seismic records is smaller. Meanwhile, it is not enough to use records about a few major earthquakes only to inspect location results of the earthquake early warning, so this thesis plans to use the historical earthquake catalog to inspect the earthquake early warning location methods. It assumes that the earthquake early warning system can pick up the same P-wave arrival information as the artificial catalog when major earthquakes occur. Because the 


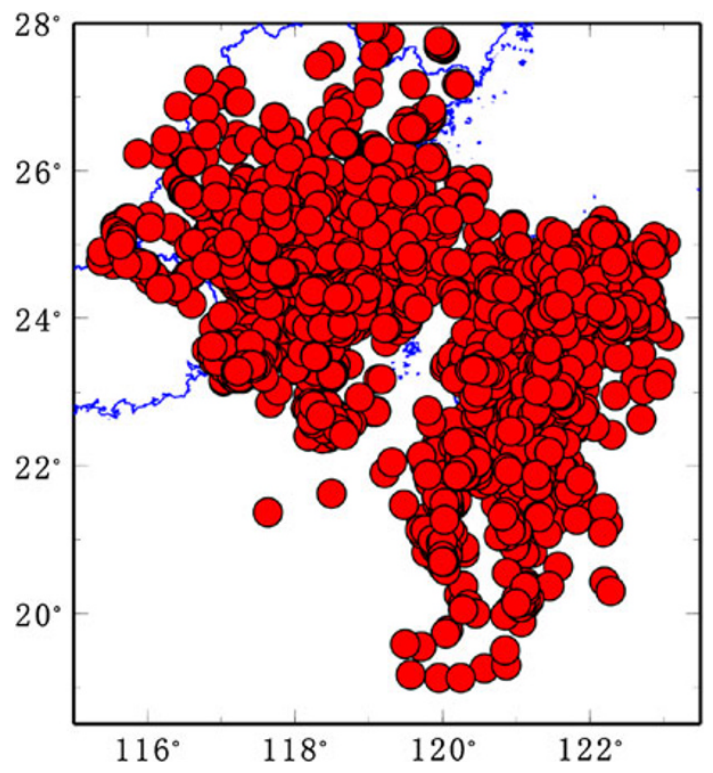

Fig. 1 Earthquake epicenter distribution

earthquake early warning mainly uses records on the near field to conduct fast and quick earthquake reports, such records on the near field cannot take the record of the clear $\mathrm{S}$-wave phase arrival information usually when major earthquake occur because of large-scale rupture. Therefore, this thesis analyzes the P-wave arrival time in the earthquake catalog.

This thesis uses the earthquake catalog information of Fujian Seismic Network in September from 1999 to 2008, selects seismic events which record the P-wave phase by at least four stations, and adopts the P-wave arrival time of the first four stations in each seismic events to inspect the two location methods. Considering the situation that the PG and $\mathrm{PN}$ phases cannot be distinguished during the process (the authors believe that the real-time processing of earthquake early warning cannot distinguish the two phases), this thesis does not distinguish the two seismic phases in the process. After screening, 5,920 seismic events are selected in all, and epicenter distribution is shown in Fig. 1.

\section{Analysis of location results}

\subsection{The analysis of location results obtained by $T_{\text {now }}$}

This thesis makes use of $T_{\text {now }}$ to carry out relocation for seismic events after screening, the search scope is less than $2,000 \mathrm{~km}$ away from the first station and the searching depth is $0-700 \mathrm{~km}$. We discover that there are 152 seismic events, whose location cannot be realized by $T_{\text {now }}$ method, taking up about $2.6 \%$ in the total number of seismic events. As the $T_{\text {now }}$ method applies information about stations without being triggered, correct triggering sequence and triggering time of stations in earthquake location have large effects on location results, and the earthquake catalog information does not include the operating state of stations. As a result, seismic events selected in the thesis cannot guarantee that all stations operate normally, because some stations failed to be reflected in earthquake catalog for discontinuous record or unclear records of seismic phases. In order to recover the operating state of seismic stations, this thesis recalculates the sequence according of stations which are theoretically triggered according to the epicenter in the earthquake catalog. In consideration of the situation that differences in medium in all directions may cause the phenomenon that stations that do not have much difference in epicentral distance may suffer reverse order, this thesis calculates the arrival time sequence of the first five stations. As a result, it is discovered that only two or less than two stations of the first five stations which are theoretically triggered by 84 of the 152 seismic events that cannot be located by $T_{\text {now }}$ method can be reflected in the earthquake catalog. The reason for this may be that operating state of these stations is abnormal or seismic phase of the P-wave is not clear because the earthquake is too small. This kind of seismic events takes the majority in the events that cannot be located. Thus, it can be seen that the main cause for failed location by $T_{\text {now }}$ method is that the order in which stations are triggered is wrong. In the actual earthquake early warning, wrong seismic phase pick-up will also lead to this kind of mistakes. In addition, some stations have abnormalities like discontinuous records, and obvious increase in signal transport delay may also cause such mistakes.

In order to analyze location results of earthquakes further, this thesis divides seismic events into earthquakes within the network and earthquakes outside the network. The former in this thesis refers to seismic events within the outside line of all stations and the number of such seismic events is 1,418 . The latter refers seismic events beyond out of the outside line of stations and the number of such seismic events is 4,502 .

With respect to earthquakes within the network, there are six seismic events that cannot be located, which only account for $0.4 \%$ in the total number of earthquakes. Epicentral deviation in the earthquake catalog is shown in Fig. 2, where $X$-axis refers to the distance from the first station to the epicenter in the earthquake catalog and $Y$-axis is the epicentral deviation. According to the results, the maximum deviation of the $T_{\text {now }}$ location method is $160 \mathrm{~km}$. Besides, positioning deviation of 991 events is less than $10 \mathrm{~km}$, taking up $70 \%$ in the total number of events. Additionally, the epicentral deviation of 1,355 seismic events is less than $30 \mathrm{~km}$, taking up $96 \%$ in the total number of events. Their average deviation is about 


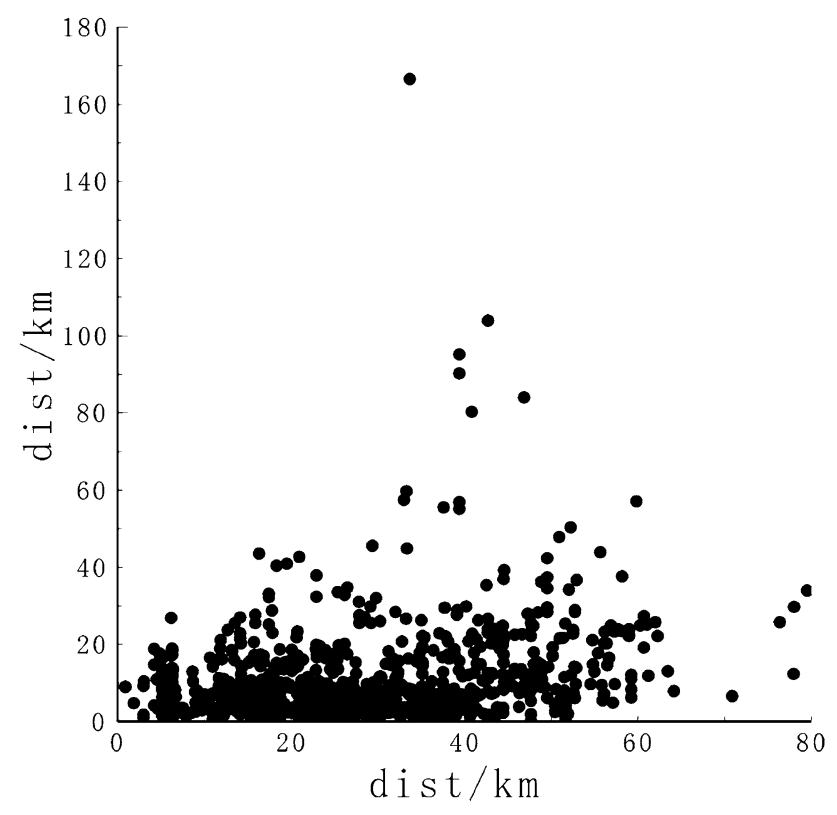

Fig. 2 Use the $T_{\text {now }}$ method to deal with earthquakes within network; $X$-axis refers to the distance from the first station to the epicenter in the earthquake catalog and $Y$-axis is the epicentral deviation

$9.4 \mathrm{~km}$ and variance is $11.4 \mathrm{~km}$. Thus, it can be seen that the deviation is relatively small when $T_{\text {now }}$ method is used for location.

With respect to earthquakes outside the network, there were 146 seismic events which cannot be located, whose number is relatively large. Epicentral deviation in the earthquake catalog is shown in Fig. 3 where $X$-axis refers to the distance from the first station to the epicenter in the earthquake catalog and $Y$-axis is the epicentral deviation. According to the results, the maximum deviation of the $T_{\text {now }}$ location method is $1,200 \mathrm{~km}$. Besides, the positioning deviation of 1,837 seismic events is less than $100 \mathrm{~km}$, taking up $42 \%$ in the total number of events. Additionally, the deviation of 270 seismic events is larger than $300 \mathrm{~km}$, taking up $6 \%$ in the total number of events. It can be said that the positioning deviation is relatively large.

In the figure, it shows that the positioning deviation may also increase as the distance from the first station to the epicenter in the earthquake catalog increases. The reason for this may be that stations are centered on one side for the epicenter. The farther the earthquake is, the smaller the opening angle between the station taking part in location and the earthquake will be. In the figure, it is also displayed that the positioning deviation of some seismic events within $200-300 \mathrm{~km}$ from the first station to epicenter is larger, but their causes are still unknown. This thesis plans to carry out detailed analysis of these seismic events.

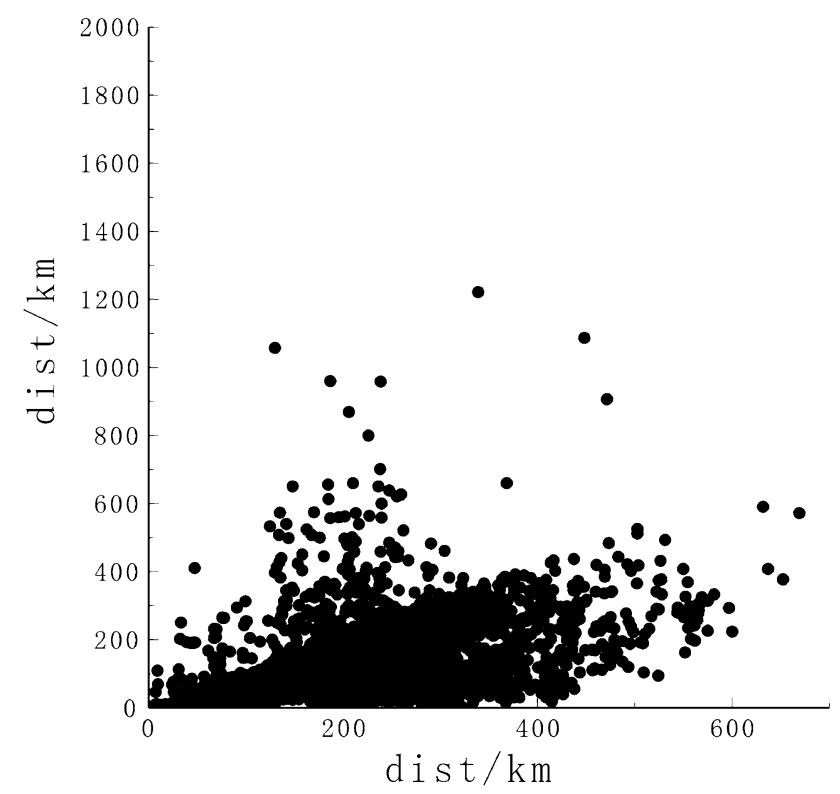

Fig. 3 Use the $T_{\text {now }}$ method to deal with earthquakes outside network; $X$-axis refers to the distance from the first station to the epicenter in the earthquake catalog and $Y$-axis is the epicentral deviation

\subsection{Analysis of location results obtained by the four- station continuous location method}

Results obtained by using the four-station continuous location method to deal with 1,418 earthquakes within network are shown in Fig. 4. In detail, there is one seismic event which cannot be located, 18 events whose positioning deviation is more than $100 \mathrm{~km}$ and 1,076 events whose positioning deviation is less than $10 \mathrm{~km}$ and that account for $76 \%$ in the total number of events. 1,355 events whose positioning deviation is less than $30 \mathrm{~km}$ and which take up $96 \%$ in the total number of events. The average deviation is $11.2 \mathrm{~km}$ and the variance is $35 \mathrm{~km}$. In general, differences in the location results obtained by the four-station continuous location method and $T_{\text {now }}$ are not obvious. However, because some seismic events have large location errors, the average deviation and variance of the overall location for the four-station continuous location method is larger compared with that of $T_{\text {now }}$.

Results obtained by using the four-station continuous location method to deal with 1,418 earthquakes outside the network are shown in Fig. 5. There are 42 events that cannot be located; 37 events whose positioning deviation is larger than $1,000 \mathrm{~km}, 1,670$ events whose positioning deviation is less than $100 \mathrm{~km}$ and which take up $37 \%$ in the total number of events and 553 events whose positioning deviation is more than $300 \mathrm{~km}$ and that take up $12 \%$ in the total number of events. Similarly, the positioning deviation of the four-station continuous location method may increase as the distance from the first station 


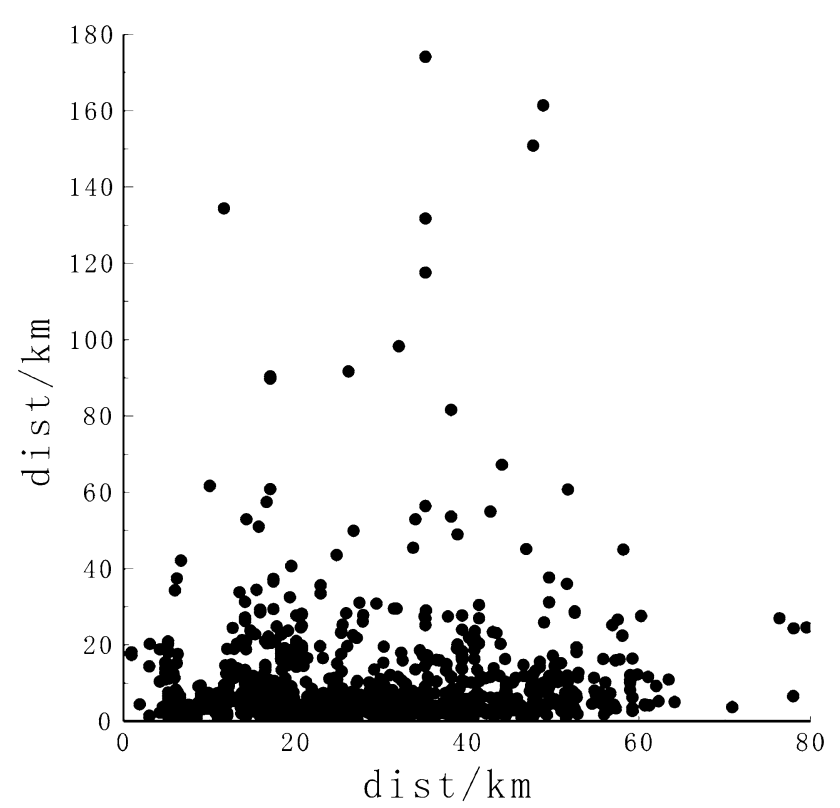

Fig. 4 Use the four-station continuous location method to deal with earthquakes within network; $X$-axis refers to the distance from the first station to the epicenter in the earthquake catalog and $Y$-axis is the epicentral deviation

to the epicenter in the earthquake catalog increases. Compared with the $T_{\text {now }}$ method, events with large positioning deviation are more when the four-station continuous location method is used, which may be related to selected P-wave velocity structure. The four-station continuous location method assumes that P-wave velocity structure is a uniform model. Whereas, $T_{\text {now }}$ method is onedimensional multilayer model (in practical application, we could select other more applicable models in the region). For earthquake within the network, the assumption that the wave velocity is locally uniform may be reasonable, while impacts of the wave velocity on the location results may be significant for earthquakes outside the network. This may result in some limitations when this method is used to deal with earthquakes outside the network. In this thesis, the $T_{\text {now }}$ location method adopts South China Model which is a one-dimensional multilayer model. In practical application, other models which are appropriate for a certain region can be chosen.

\subsection{Comparison between the two location methods}

In order to compare the location results obtained by the two location methods, we make a comparative analysis of the location results of the seismic events which can be located by two methods. As shown in Table 1, the conclusion indicated that the location results of two methods are relatively similar for earthquakes within the network. Most of the positioning deviations are less than $30 \mathrm{~km}$, while

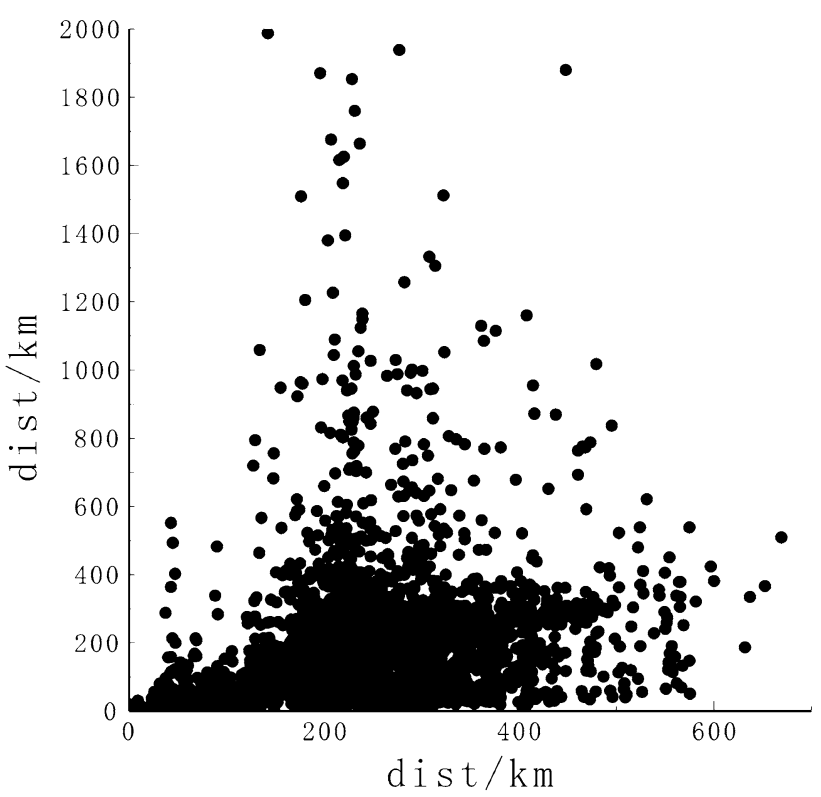

Fig. 5 Use the four-station continuous location method to deal with earthquakes outside network; $X$-axis refers to the distance from the first station to the epicenter in the earthquake catalog and $Y$-axis is the epicentral deviation

seismic events whose positioning deviations is more than $50 \mathrm{~km}$ when the four-station continuous location method is used are more compared with $T_{\text {now }}$, which might relate to several denominators in equations that are close to zero when the four-station continuous location method is applied. For earthquakes outside network, the number of seismic events whose positioning deviation is less than $100 \mathrm{~km}$, which are located by the two methods, is relatively similar. However, the number of seismic events whose deviations is less than $200 \mathrm{~km}$ and which are located by the $T_{\text {now }}$ method is a little more. The reason for this may be that $T_{\text {now }}$ uses location information of the stations which are not triggered so that there are more constraints for the epicenter.

\section{Conclusions}

Based on records in the earthquake catalog of Fujian Seismic Network, seismic events are selected which have recorded at least four P-wave Seismic phases. The thesis uses the P-wave arrival information of the first four stations in each seismic event to test the $T_{\text {now }}$ location method and the four-station continuous location method put forward by Jin Xing. As a result, it is found that the number of seismic events which cannot be located by the $T_{\text {now }}$ method is more than that for the four-station continuous location method. According to the results of the earthquake catalog location, this thesis recalculates theoretical triggering 
Table 1 Location results in the two seismic events that both could be positioned

\begin{tabular}{|c|c|c|c|c|c|c|}
\hline \multirow[t]{2}{*}{ Positioning deviation } & \multicolumn{3}{|c|}{$\begin{array}{l}\text { Earthquake within network } \\
(\mathrm{km})\end{array}$} & \multicolumn{3}{|c|}{$\begin{array}{l}\text { Earthquake outside network } \\
(\mathrm{km})\end{array}$} \\
\hline & $<10$ & $<30$ & $>50$ & $<100$ & $<200$ & $>300$ \\
\hline The number of earthquakes ( $T_{\text {now }}$ method) & 990 & 1,354 & 17 & 1,822 & 3,103 & 264 \\
\hline Proportion in the total number of events $(\%)$ & 70.2 & 96.0 & 1.2 & 42.3 & 72.0 & 6.1 \\
\hline The number of earthquakes (the four-station continuous location method) & 1,074 & 1,350 & 33 & 1,613 & 2,704 & 524 \\
\hline Proportion in the total number of events $(\%)$ & 76.1 & 95.7 & 2.3 & 37.4 & 62.8 & 12.2 \\
\hline
\end{tabular}

sequence of seismic stations. It shows that, among the majority of seismic events which cannot be located by the $T_{\text {now }}$ method, the first five theoretically triggered seismic stations are not coincident with the four stations used to locate, or only have a few coincidences (less than three stations). The reason for this may be that the operating state of these stations is abnormal so that $\mathrm{P}$-wave seismic phase cannot be identified or P-wave seismic phase is unclear because the earthquakes are too small. Therefore, the correct triggering sequence and triggering time of seismic stations have a great impact on the location results obtained by the $T_{\text {now }}$ location method in real seismic events.

For earthquakes whose positioning results can be given, the differences in the location results of the two methods are not significant and the positioning deviation of most seismic events is small. When denominators in equations are zero or close to zero when the four-station continuous location method is applied, the positioning deviation may be large. For earthquakes within the network, impacts of a wave velocity model on the location results are relatively small because stations surround the epicenter well. However, for earthquakes outside the network, since all seismic stations spread toward one side of the epicenter, selecting a velocity model which is similar to actual structure of the wave velocity will contribute to improving location results of earthquakes.

For the real earthquake early warning, the limitations of the two location methods should be taken into account comprehensively. For example, using the $T_{\text {now }}$ method for earthquake early warning location, we need to set a high requirement, that is, the system should quickly identify the running state of seismic stations and reject information about seismic stations with abnormal operating status and discontinuous records (e.g., interruption logger, etc.) to monitor the seismic stations running state in real time. Meanwhile, network delay and other problems also affect the application of the $T_{\text {now }}$ method. In general, using the $T_{\text {now }}$ method, we need to set a certain time to deal with network delay, while the specific time of network delay may be uncertain in real network. Especially, when the majority earthquake came, the quantity of data transmission increases abruptly, and other data in the network will probably increase obviously, which influences the transmission speed to a certain extend.
Moreover, the four-station continuous location methods merely take advantage of the arrival information from the triggered station, that is, abnormalities including the network delay and the interruption logger have little influence on the location results. Nevertheless, this method does not use the information of other stations without being triggered and has a little restriction on the epicenter. Especially, when the denominators of equations calculated by this method equal 0 or approach 0 , large positioning deviation may appear and even unreasonable epicenter position will occur. The assumption that the wave velocity is uniform may also lead to some limitations when the two methods are used to deal with earthquakes outside the network. Comprehensively taking the results of the two methods above into account and screening the data may contribute to enhancing the reliability and stability of earthquake early warning systems.

In earthquake early warning systems, since the two methods apply the same seismic phase arrival time, the time that the two take to give location results does not have significant difference. In order to ensure the reliability of early warning information output in the system and minimize the risk of false alarms, we may also need to consider whether the parameters including travel time residuals, the location of the epicenter, amplitude, predominant period, and real-time instrument intensity match with one another.

\section{References}

Hasegawa A, Umino N, Takagi N (1978) Double-planedstructure of the deep seismic zone in the northeastern Japan Arc. Tectonophysics $47: 43-58$

Horiuchi S, Negishi H, Abe K et al (2005) An automatic processing system for broadcasting earthquake alarms. Bull Seismol Soc Am 95(2):708-718

Jin X, Zhang HC, Li J et al (2012) Reserch on continus location method used in the earthquake early warning system. Chin J Geophys 55(3):925-936 (in Chinese)

Nakamura Y (1988) On the urgent earthquake detection and alarm system (UrEDAS). In: Proceedings of Ninth World Conference on Earthquake Engineering, vol 7, pp 673-678

Nakamura Y (1989) Earthquake alarm system for Japan Railways. Japn Railw Eng 109:1-7

Nakamura Y, Tucker BE (1988) Japan's earthquake warning system: should it be imported to California? Calif Geol 41(2):33-40 
Odaka T, Ashiya K, Tsukada S et al (2003) A new meth epicentral distance and magnitude from a single seismic. Bull Seismol Soc Am 93(1):526-532

Rydelek P, Pujol J (2004) Real-timeseismic warning with a 2-station subarray. Bull Seismol Soc Am 94:1546-1550
Satriano C, Lomax A, Zollo A (2008) Real-time evolutionary earthquake location for seismic early warning. Bull Seismol Soc Am 98:1482-1494

Wu YM, Teng T (2002) A virtual subnet work approach to earthquake early warning. Bull Seismol Soc Am 92(5):2008-2018 